\title{
Tailoring cations in a perovskite cathode for proton-conducting solid oxide fuel cells with high performance
}

\author{
$\mathrm{Xi} \mathrm{Xu}{ }^{\text {a\#}}$, Huiqiang Wang ${ }^{\mathrm{b}}$, Marco Fronzi ${ }^{\mathrm{c}}$, Xianfen Wang ${ }^{\mathrm{a}}$, Lei Bi ${ }^{\mathrm{a}}{ }^{*}$ and Enrico \\ Traversa $^{\text {d* }}$
}

a. Institute of Materials for Energy and Environment, College of Materials Science and Engineering, Qingdao University, Ningxia Road No.308, Qingdao 266071, China.

Email: bilei@qdu.edu.cn and bilei81@gmail.com (L.Bi)

b. College of Mechanical and Electric Engineering, Hebei Agricultural University, Baoding 071001, Hebei, China

c. International Research Centre for Renewable Energy, State Key Laboratory of Multiphase Flow in Power Engineering, Xi'an Jiaotong University, Xi'an, China

d. School of Materials and Energy, University of Electronic Science and Technology of China, 2006 Xiyuan Road, Chengdu 611731, Sichuan, China. Email: traversa@uestc.edu.cn (E.Traversa)

\#: Xi Xu and Huiqiang Wang contributed equally to this paper.

*: Corresponding authors 


\section{Abstract}

A rational design of a high-performance cathode for proton-conducting solid oxide fuel cells (SOFCs) is proposed in this study with the aim of improving the hydration properties of conventional perovskite cathode materials, thus leading to the development of new materials with enhanced proton migration. Herein, potassium is used to dope traditional $\mathrm{Ba}_{0.5} \mathrm{Sr}_{0.5} \mathrm{Co}_{0.8} \mathrm{Fe}_{0.2} \mathrm{O}_{3-\delta}$ (BSCF), which is demonstrated to be a beneficial way for improving hydration, both experimentally and theoretically. The theoretical study was needed to overcome practical limits that hindered direct hydrogen mobility measurements. The novel material $\mathrm{Ba}_{0.4} \mathrm{~K}_{0.1} \mathrm{Sr}_{0.5} \mathrm{Co}_{0.8} \mathrm{Fe}_{0.2} \mathrm{O}_{3-\delta}$ (BKSCF) shows a lower overall proton migration energy compared with that of the sample without K, suggesting that K-doping enhances proton conduction, which shows an improved performance by extending the catalytic sites to the whole cathode area. As a result, a fuel cell built with the novel BKSCF cathode shows an encouraging fuel cell performance of 441 and $1275 \mathrm{~mW} \mathrm{~cm}$ at 600 and $700{ }^{\circ} \mathrm{C}$, respectively, which is significantly higher than that of the cell using the pristine BSCF cathode. This study provides a new and rational way to design a perovskite cathode for proton-conducting SOFCs with high performance. 


\section{Introduction}

The world is currently undergoing an energy crisis, and solid oxide fuel cells (SOFCs) can be used to mitigate this problem, as they can efficiently convert chemical energy into electricity without involving the combustion process that traditional methods have to use. ${ }^{1,2}$ Currently, research on SOFCs using proton-conducting electrolytes has become a hot topic in the community, as proton-conducting SOFCs (H-SOFCs) can lower the working temperature of SOFCs to the intermediate range, thus extending the SOFC lifetime. ${ }^{3-5}$ In addition, no fuel dilution occurs at the fuel electrode for H-SOFCs. ${ }^{6}$ However, with the decrease in working temperature, cathode polarization resistance dramatically increases, and it is generally believed that the cathode catalytic activity decreases with the decreasing operational temperatures. ${ }^{7}$ However, this is not the whole landscape. It should be noted that many high-performance cathode materials for oxygen-ion conducting SOFCs (O-SOFCs), each with excellent catalytic activity at intermediate temperatures, have been proposed for H-SOFCs, ${ }^{8}$ but their performance is not satisfactory. The cathode reaction mechanisms revealed that conduction of protons, oxygen-ions and electrons are all important for the cathode performance of $\mathrm{H}-\mathrm{SOFCs},{ }^{9}$ while only oxygen-ion and electron conductions are involved in oxygen-ion conducting SOFCs, ${ }^{10}$ which could explain the unsatisfactory performance of existing O-SOFC cathodes used for H-SOFCs. In other words, the creation of improved proton conduction in the 
cathode materials is a rational route for designing proper cathodes for H-SOFCs. ${ }^{11-13}$

$\mathrm{Ba}_{0.5} \mathrm{Sr}_{0.5} \mathrm{Co}_{0.8} \mathrm{Fe}_{0.2} \mathrm{O}_{3-\delta}$ (BSCF) is a highly catalytic cathode at intermediate temperatures for O-SOFCs, and its catalytic activity is even more remarkable at low temperatures. ${ }^{14}$ Some recent work suggests that BSCF has a proton-conduction potential due to its hydration ability, ${ }^{15-17}$ which implies that, in principle, BSCF should behave properly in H-SOFCs. However, previous studies demonstrated that BSCF only shows an average performance for H-SOFCs. ${ }^{18}$ The performance of H-SOFCs using a BSCF cathode is not as impressive as when the cathode is used for O-SOFCs. It is known that BSCF shows high oxygen-ion conduction with sufficient electronic conduction; ${ }^{19}$ therefore, proton-conduction might be the rate-limiting step when used in H-SOFCs. Although the potential proton-conducting behaviour of BSCF is demonstrated by its hydration ability, ${ }^{17}$ proton-conduction might not be sufficient, and the improvement of proton migration in BSCF would be a rational route for the design of BSCF-based cathodes for H-SOFCs. Protonic defects can be stabilized in oxides with strong basicity, ${ }^{20,21}$ so it is reasonable to assume that the proton migration in BSCF could be enhanced by increasing the basicity of the material, thus enhancing its catalytic activity for the cathode.

Inspired by this idea, potassium is used here to partially replace barium in BSCF, with the aim to improve hydration and proton migration. Both experimental studies and theoretical calculations are performed to investigate the properties of the new materials. In addition, a full fuel cell was fabricated and tested to demonstrate the 
suitability of the BKSCF new material for H-SOFCs.

\section{Experimental}

Both $\mathrm{Ba}_{0.5} \mathrm{Sr}_{0.5} \mathrm{Co}_{0.8} \mathrm{Fe}_{0.2} \mathrm{O}_{3-\delta}$ (BSCF) and $\mathrm{Ba}_{0.4} \mathrm{~K}_{0.1} \mathrm{Sr}_{0.5} \mathrm{Co}_{0.8} \mathrm{Fe}_{0.2} \mathrm{O}_{3-\delta}$ (BKSCF) powders were synthesized by a combustion method ${ }^{22}$ using $\mathrm{Ba}\left(\mathrm{NO}_{3}\right)_{2}, \operatorname{Sr}\left(\mathrm{NO}_{3}\right)_{2}$, $\mathrm{Co}\left(\mathrm{NO}_{3}\right)_{3}$ and $\mathrm{Fe}\left(\mathrm{NO}_{3}\right)_{3}$ as the starting materials. The $\mathrm{K}$ source was obtained by dissolving $\mathrm{K}_{2} \mathrm{CO}_{3}$ in nitric acid to form $\mathrm{KNO}_{3}$. The phase purity of the as-prepared powders after firing at $950^{\circ} \mathrm{C}$ for $3 \mathrm{~h}$ was examined by using X-ray diffraction $(\mathrm{XRD}$, Rigaku Ultima IV) analysis. High-resolution transmission electron microscopy (HRTEM, JEM-2100F) was used to measure the spacing of lattice fringe of the as-prepared BSCF and BKSCF samples. Hydration tests were carried out by hydrating the BSCF and BKSCF samples at $200{ }^{\circ} \mathrm{C}$ with flowing wet air for $24 \mathrm{~h}$, and then the water contents of the hydrated samples were measured by the Karl-Fischer titration method using Titrando KF equipment (Metrohm) coupled with a furnace. X-ray photoelectron spectroscopy (XPS) analysis of both BSCF and BKSCF powders was conducted on a Thermo Fisher ESCALAB 250Xi spectrometer using an AlKa (1486.6 eV) radiation source. To evaluate the chemical stability of BSCF and BKSCF, both samples were treated in $3 \% \mathrm{CO}_{2}$ atmosphere (balanced with $\mathrm{Ar}$ ) at $650{ }^{\circ} \mathrm{C}$ for $2 \mathrm{~h}$ and then temperature Programmed Desorption (TPD, Quantachrome Chembet Pulsar analyzer) tests were performed on $\mathrm{CO}_{2}$ pre-treated $\mathrm{BSCF}$ and $\mathrm{BKSCF}$ powders to examine their desorption behaviors. In addition, the BSCF and BKSCF powders before and after the $\mathrm{CO}_{2}$ treatment were also characterized by Fourier-transform 
infrared spectroscopy (FTIR, Thermo Scientific iS50 FT-IR) to check the formation of carbonates.

BKSCF was tested as the cathode for $\mathrm{H}-\mathrm{SOFCs}$ with $\mathrm{BaCe}_{0.7} \mathrm{Zr}_{0.1} \mathrm{Y}_{0.2} \mathrm{O}_{3-\delta}(\mathrm{BCZY})$ electrolytes. The BCZY powder was synthesized by a combustion method, and the preparation details can be found elsewhere. ${ }^{23}$ The anode powders were prepared by mechanically mixing commercial $\mathrm{NiO}$ and $\mathrm{BCZY}$ powders in a 6:4 weight ratio, adding $20 \mathrm{wt} \%$ starch as the pore former. For the anode functional layer, $\mathrm{NiO}$ and BCZY (in a 6:4 weight ratio) powders were prepared using a conventional combustion method. ${ }^{24}$ Then, NiO-BCZY(anode)/ NiO-BCZY (functional layer)/BCZY(electrolyte) half-cells were prepared by co-pressing, followed by sintering at $1300{ }^{\circ} \mathrm{C}$ for $6 \mathrm{~h}$. The diameter of the sintered half-cell was around $12 \mathrm{~mm}$. Finally, a BKSCF(70 wt.\%)-BCZY(30 wt.\%) composite cathode slurry was deposited on the surface of the sintered BCZY electrolyte membrane, thus completing the cell by annealing at $900{ }^{\circ} \mathrm{C}$. For comparison, identical cells were prepared using cathode pristine BSCF powders instead of BKSCF. Fuel cell tests were performed using wet $\mathrm{H}_{2}$ as the fuel and static air as the oxidant. The I-V curves and electrochemical impedance spectroscopy (EIS) measurements of the cells were recorded using an electrochemical workstation (CHI604E and CHI1110C, CH Instrument). The cell morphology was observed using a scanning electron microscope (SEM, JSM-7800F).

All calculations were performed using the density functional theory (DFT), ${ }^{25}$ as was implemented in the VASP (Vienna ab initio simulation package) ${ }^{26,}{ }^{27}$ with the 
Perdew, Burke and Ernzerhof $(\mathrm{PBE})^{28}$ exchange correlation potential within the generalized gradient approximation $(\mathrm{GGA})^{29}$. The projector augmented wave (PAW) pseudopotential wave was used to describe core electrons. ${ }^{30}$ For Hubbard's correction, ${ }^{31,32}$ the $\mathrm{U}_{\text {eff }}$ value was set to $4 \mathrm{eV}$ and $3.32 \mathrm{eV}$ for $\mathrm{Fe}$ and $\mathrm{Co}$, respectively. ${ }^{33}$ For all optimization calculations, the convergence criteria for energy and force were set to $10^{-5} \mathrm{eV}$ and $0.03 \mathrm{eV} \AA^{-1}$, respectively. The cutoff energy was set to $520 \mathrm{eV}$, and a $4 \times 4 \times 4 \mathrm{~K}$-point mesh gamma centred was chosen to describe the Brillouin zone. To analyse proton migration, we used a climbing-image nudged elastic band (CI-NEB) method, ${ }^{34}$ which allowed us finding the migration barriers when the convergence criteria for energy and force were set to $10^{-4} \mathrm{eV}$ and $0.05 \mathrm{eV}$ $\AA^{-1}$, respectively.

\section{Results and Discussion}

Figure 1 shows the XRD patterns of the BKSCF and the pristine BSCF powders as a reference. The BKSCF powder showed peaks of the perovskite structure, without detectable impurities. By comparing the reflection peaks of BKSCF and BSCF (Fig. $1 \mathrm{~b}$ for the (211) peak), one can observe that the BKSCF peaks shifted slightly towards lower angles. The calculated lattice parameter for BSCF is $3.967 \AA$, while the value for BKSCF is $3.985 \AA$, which suggests an expansion of the lattice parameter with $\mathrm{K}$-doping. This is probably due to the incorporation of $\mathrm{K}$ into the lattice, because of a larger ionic radius of $\mathrm{K}^{+}\left(164 \mathrm{pm}\right.$ at 12 -coordinate) compared with that of $\mathrm{Ba}^{2+}(161$ 
pm at 12-coordinate). ${ }^{35} \mathrm{~A}$ larger spacing value for the lattice fringe corresponding to the (211) planes is obtained for BKSCF compared with that for BSCF that is observed from HRTEM (Figure S1), further confirming the lattice expansion when $\mathrm{K}$ is used to replace $\mathrm{Ba}$. The defect chemical reaction could be written as follows:

$$
\mathrm{K}_{2} \mathrm{O} \stackrel{\mathrm{BaO}}{\longrightarrow} 2 \mathrm{~K}_{\mathrm{Ba}}^{\prime}+\mathrm{O}_{o}^{x}+V_{o}^{\bullet \bullet}
$$

As the original idea of doping $\mathrm{K}$ into $\mathrm{BSCF}$ is to create improved proton-conduction in the new material due to the high basicity of $\mathrm{K}$ and thus to enhance the cathode catalytic activity for H-SOFCs, the possible proton conduction in the material in comparison with the conventional K-free BSCF was investigated. The accepted proton formation mechanism is through the incorporation of $\mathrm{H}_{2} \mathrm{O}$ into oxygen vacancies $\left(V_{o}^{* *}\right)$, which creates proton charge carriers, ${ }^{36}$ as shown in the equation below:

$$
\mathrm{H}_{2} \mathrm{O}+\mathrm{V}_{o}^{\bullet \cdot}+\mathrm{O}_{o}^{\times} \Leftrightarrow 2 \mathrm{OH}^{\bullet}
$$

One can see that the fundamental prerequisite for proton formation and subsequent proton migrations in the lattice is the presence of oxygen vacancies. To have an insight into this mechanism, we performed DFT calculations to predict the change of oxygen vacancy formation energy with K-doping. Oxygen vacancy formation energy values were calculated considering the molecular oxygen in the gas phase according to the following equation: ${ }^{36}$

$$
E_{f}=E_{\text {def ect }}+\frac{1}{2} E_{Q}-E_{\text {perf er }}
$$

where $E_{f}$ is the oxygen vacancy formation energy; $E_{\text {def ect }}$ is the total energy of 
defective bulk, where one $\mathrm{O}$ atom was removed from 40 -atoms bulk; $E_{\mathrm{O}_{2}}$ is the energy of molecular oxygen; and $E_{\text {perf ecl }}$ is the total energy of the stoichiometric bulk. We considered two types of oxygen vacancy in the BSCF and BKSCF lattices, as shown in Figure 2, where one is $\mathrm{Co}-V_{o}^{\cdot \bullet}$-Co and the other is Fe- $V_{o}^{\cdot \bullet}$-Co. There is a total of $24 \mathrm{O}$ atoms, all bonded to both $\mathrm{Ba}$ and $\mathrm{Sr}$ atoms. Oxygen vacancy formation energy was calculated by removing one oxygen atom from the stoichiometric bulk. Therefore, there are a total of 24 possible vacancy sites. Also, each oxygen vacancy site is connected to Fe and Co. Here we analyze Co and Fe doping at different concentration. Therefore, the $\mathrm{Fe}-\mathrm{O}$ and $\mathrm{Co}-\mathrm{O}$ bonds differ in number and binding energy. In view of this, we consider the following vacancy configurations: $C o-V_{o}^{*}$ - $\mathrm{Co}$ and $\mathrm{Fe}-\mathrm{V}_{o}^{\cdot \bullet}$-Co. Additional configurations are also considered, taking into account the influence of the second nearest TMs. Here, there are two Co and two Fe ions surrounding $\mathrm{Co}-\mathrm{V}_{o}^{\cdot}{ }^{-} \mathrm{Co}$, four Co ions surrounding $\mathrm{Fe}-V_{o}^{\cdot \bullet}$-Co. In BKSCF, K doped at $\mathrm{Ba}$ site, only $\mathrm{O}$ vacancy position around $\mathrm{K}$ was considered, since it is the configuration associated with the structure that shows a better catalytic activity. The better catalytic is demonstrated by the calculated better vacancy formation ability and hydration ability. Table 1 shows the calculated oxygen vacancy formation energy of Co- $V_{o}^{*}$-Co and $\mathrm{Fe}-V_{o}^{\bullet}$-Co in BSCF and BKSCF, respectively.

Our results show that the oxygen vacancy formation in both pristine and doped material is an exothermic reaction, which suggests that the formation of oxygen vacancy is thermodynamically favourable for both materials, in agreement with the 
literature. ${ }^{37}$ A relatively small difference between $\mathrm{Co}-V_{o} \cdot{ }^{\cdot}$-Co and $\mathrm{Fe}-V_{o}^{*}$-Co oxygen vacancy formation energy values was found for BSCF, calculated at $1.189 \mathrm{eV}$ and $1.211 \mathrm{eV}$, respectively. These values are similar to that reported for $\mathrm{BSCF}$ in the previous report. ${ }^{37}$ Although the oxygen vacancy in Fe- $V_{o}^{*}$-Co type is slightly favourable, its proportion in the BSCF bulk material is much lower than that for Co$V_{o}^{*}$-Co.

Since an easier oxygen vacancy formation is expected in BKSCF, owing to the lowest oxygen formation energy when $\mathrm{Ba}$ is replaced by $\mathrm{K}$ ions, to simplify computation for $V_{o}^{*}$ in $\mathrm{BKSCF}$, we considered only properties around $\mathrm{K}$. The results showed that BKSCF has a higher oxygen vacancy formation ability for both vacancy types: the values were calculated at $1.69 \mathrm{eV}$ and $1.51 \mathrm{eV}$ in $\mathrm{Co}-V_{o}^{*}$-Co and Fe- $V_{o}^{*}$-Co, respectively, suggesting that $\mathrm{K}$-doping in BSCF facilitates oxygen vacancy formation. We found a clear trend in K-doping to form $V_{o}^{\cdot \bullet}$ due to the charge compensation that is needed when $\mathrm{K}$ is doped in the material. The large $\mathrm{K}$ ionic radius also provides a high steric hindrance to decrease the truncation metal-oxygen bond, thereby leading to a lower oxygen vacancy formation energy for the BKSCF sample. It is also worth noting that the K-doping impact on the oxygen vacancy formation energy is larger for the $\mathrm{Co}-V_{o}^{*}$-Co than for the Fe- $V_{o}^{*}$-Co type, which makes the former oxygen vacancy type more favourable in the material than Fe- $V_{o}^{\cdot \bullet}$-Co. The energy difference in the oxygen vacancy formation in the K-doped oxide suggests a higher oxygen vacancy density in BKSCF with respect to BSCF. 
For cathodes working in H-SOFCs, the hydration ability is essential for the electrochemical performance. When the basicity of the material is stronger, the hydration in principle becomes easier. In the case of our study, the partial replacement of $\mathrm{Ba}$ by $\mathrm{K}$ in the material could lead to stronger basicity for BKSCF compared with BSCF, due to the high basicity of K. Therefore, an improved hydration ability and thus a high proton uptake could be expected for BKSCF. The hydration energy, which represents the feasibility of hydration for a given material, was also calculated using the DFT method, according to equation (4): ${ }^{38}$

$$
\Delta E_{\text {hydration }}=E_{2 \mathrm{OH}}-E_{\text {def ect }}-E_{H \mathrm{C} C}
$$

where $\Delta E_{\text {hydration }}$ is the hydration energy, $E_{2 O H}$ is the energy of the crystal with two additional protons, $E_{\text {def ect }}$ is the total energy of the defective bulk, and $E_{\mathrm{H}_{2} \mathrm{O}}$ is the energy of a single water molecule in the vacuum. The results are shown in Table 2.

All the calculated hydration energy values were negative, which indicated an exothermic reaction for the hydration process for both BSCF and BKSCF. The hydration energy for BKSCF increased from -0.04 to $-0.21 \mathrm{eV}$ for $\mathrm{Co}-V_{o}^{*}-\mathrm{Co}$, and from -0.21 to $-0.3 \mathrm{eV}$ for $\mathrm{Fe}-V_{o}^{*}-\mathrm{Co}$. This finding suggests that K-doping improves the hydration ability for both $\mathrm{Co}-V_{o}^{\cdot \bullet}$ - $\mathrm{Co}$ and $\mathrm{Fe}-V_{o}^{\cdot \bullet}$-Co types, which makes the impact of K-doping for hydration more evident for Co- $V_{o}^{\cdot \bullet}$-Co than for Fe- $V_{o}^{\cdot \bullet}$-Co, in agreement with the results that were obtained for oxygen formation energy. Since Co$V_{o}^{*}$-Co is the more abundant vacancy type in the material, it is reasonable to predict that the hydration ability improves in BKSCF with respect to pristine BSCF. 
To validate the results from the above theoretical calculations, the hydration behavior for BKSCF and BSCF was evaluated experimentally using the Karl-Fischer titration method. Both BSCF and BKSCF samples were hydrated at low temperatures, and the hydration of these oxides is expected to take place mainly through the dissociative adsorption of $\mathrm{H}_{2} \mathrm{O}$, according to equation (1). The samples were placed in a furnace which was heated to a high temperature to release the water in the material and then the released $\mathrm{H}_{2} \mathrm{O}$ was measured by the titration method. The measured water content for $\mathrm{BSCF}$ and $\mathrm{BKSCF}$ is $0.34 \%$ and $0.61 \%$, respectively, suggesting a higher proton uptake for BKSCF in comparison with the sample without K-doping. The higher water uptake for $\mathrm{K}$ doped BSCF sample is expected due to the higher basicity of $\mathrm{K}$ compared with $\mathrm{Ba}$. This finding is also consistent with the theoretical calculations. Thus, it is possible to conclude that K-doping into BSCF allows for more protons to be incorporated into the lattice when compared with that of the pristine material. This may result in an improvement of the proton migration for the BKSCF sample.

Even though BKSCF was proven to be able to hold more protons than BSCF, the mobility of these protons needs to be assessed, since mobility is the critical parameter for determining the cathode performance for H-SOFCs. It should be noted that BSCF-based material is a mixed electronic-ionic conductor in which electronic conduction is a few orders of magnitude larger than the ionic conductivity, ${ }^{39-41}$ making the ionic conductivity negligible compared with its electronic conductivity. 
Therefore, the characterization of specific proton conduction in BSCF is not feasible by conventional conductivity measurements, and one can only obtain the value of electronic conductivity for this type of materials by a direct conductivity measurement of the sample. Although some testing methods, such as hydrogen permeation ${ }^{42}$ or $\mathrm{H}_{2}$ concentration cells ${ }^{43}$, could be possible to allow separating proton conduction for the mixed conductors, they need the sample to be tested in $\mathrm{H}_{2}$-containing atmospheres. However, BSCF-based materials are not stable in reducing atmospheres, so it is difficult to characterize the specific proton conductivity in BSCF-based materials by using methods such as hydrogen permeation or direct electrochemical measurements in hydrogen. Although Hebb-Wagner method be able to measure the ionic conductivity for a mixed conductor and it has been employed for measure the oxygen-ion conductivity for mixed oxygen-electron conductors, ${ }^{44,45}$ electron-blocking layers that could allow the transportation ions and block electrons has to be used in this measurement. To measure the proton conductivity of a mixed conductor, the blocking layer has to be a proton conductor and the layer has to be dense enough without interfacial reaction with the testing mixed conductor. However, this requirement seems difficult for proton-conductors. It is known that the sinterability for proton conductors (such as $\mathrm{BaCeO}_{3}$ and $\mathrm{BaZrO}_{3}$ ) is poor, usually needing a high temperature $\left(1400\right.$ to $\left.1800{ }^{\circ} \mathrm{C}\right)$ to make the material dense, ${ }^{3,4}$ while this temperature is too high to avoid interfacial reactions between the proton conductors with the testing mixed conductors. Although some physical deposition methods (such as pulsed laser 
deposition) could be used to fabricate the blocking layer at a low temperature, the small-scale application feature of pulsed laser deposition as well as the poor chemical stability of $\mathrm{BaCeO}_{3}$ and the high resistive $\mathrm{BaZrO}_{3}$ could still make the use of Hebb-Wagner method difficult. Therefore, no such measurement is performed for proton conductivity until now. DC open circuit potential method is another method which could be used for measuring the ionic conduction of a mixed conductor. However, it is only suitable for the samples with a high ionic transference number, ${ }^{46}$ which is not the case in our study. In addition, the good control of thermodynamic conditions at each side of the two electrodes for the measurement is sometimes technically challenging. ${ }^{46}$ Therefore, the direct measurement of specific proton conduction in mixed electronic-ionic conductors is still a great challenge in the community, and in given conditions no feasible experimental method is available. Therefore, to avoid this problem and to have an understanding about the effect of K-doping on proton mobility, DFT calculations, which have been demonstrated to be a powerful tool for predicting proton migration in oxides in many previous studies, ${ }^{38}$, ${ }^{47-50}$ were used to investigate and compare proton migration in BSCF and BKSCF. Figure 3 shows a schematic of the proton migration process, which involves a hopping step and a rotating step. ${ }^{20}$

The K-doped sites could influence the migration energy of both hopping and rotating mechanisms. Table 3 shows the results of the calculated migration energy values. Our results showed that the proton hopping migration barrier is higher for 
BKSCF than for BSFC, with values of $0.34 \mathrm{eV}$ (BKSFC) and $0.22 \mathrm{eV}$ (BSFC), which suggest that K-doping makes the hopping mechanism slower. This is probably due to the larger $\mathrm{K}^{+}$radius size that expands the overall lattice and increases the TM-O bond length, thus leading to a longer pathway for proton hopping and, therefore, a larger migration energy. In contrast, the energy for the rotating step decreases in the K-doped lattice. The barrier energy dramatically decreased from $0.33 \mathrm{eV}$ to $0.07 \mathrm{eV}$ for BKSCF, which suggests that the rotating step is faster in BKSCF compared to that of BSCF. Since the rotation step does not include the proton migration from one atom to another, a larger bond length will not result in a longer pathway for proton rotation. In contrast, the expanded lattice might provide more space for the proton rotation, thus lowering the barrier energy. Proton migration consists of two main steps, hopping and rotation, which suggest that protons have to undergo two steps for its migration. Although the highest energy barrier is similar for BSCF and BKSCF, and it could represent the bottleneck of the process, the energy barrier for the second step is significantly lower in BKSCF then BSCF, which may contribute to making the proton migration more feasible in the overall process. This is a crucial finding for the improvement of cathode catalytic activity.

In addition to hydration ability and proton conduction, the oxygen reduction process is another key factor for the cathode reaction, and XPS measurements were used to analyse the oxygen species for BSCF and BKSCF. Figure 4 shows the O1s XPS peaks measured for BSCF (Fig. 4a) and BKSCF (Fig. 4b). The O1s curve was 
deconvoluted into three peaks that indicate surface oxygen transformations. The peaks at approximately $532.5 \mathrm{eV}, 531 \mathrm{eV}$ and $529 \mathrm{eV}$ correspond to oxygen in the hydroxyl group, adsorbed oxygen species and lattice oxygen, respectively. In general, oxygen species transformed on the surface according to the following steps:

$$
\mathrm{O}_{2} \leftrightarrow \mathrm{O}_{2} \stackrel{+e^{-}}{\longrightarrow} \mathrm{O}_{2}^{-} \stackrel{+e^{-}}{\longrightarrow} \mathrm{O}_{2}^{2-} \leftrightarrow 2 \mathrm{O}^{-} \stackrel{+2 e^{-}}{\longrightarrow} 2 \mathrm{O}^{2-}(\text { Lattice })
$$

It has been reported that the ratio between the adsorbed oxygen and lattice oxygen reflects the ORR catalytic ability of the cathode; the larger the ratio, the better the ORR catalytic activity. ${ }^{51}$ The calculated ratio increased from 2.7 for BSCF to 3 BKSCF, which suggested that the K-doped material may have a better catalytic activity than that of pristine BSCF. Our results are consistent with a previous study that $\mathrm{K}$ is used to dope $\mathrm{SrFe}_{\mathrm{x}} \mathrm{Co}_{1-\mathrm{x}} \mathrm{O}_{3}$-based cathode materials for improving their electrocatalytic activity for O-SOFCs. ${ }^{52}$ In addition, comparing the binding energy of lattice oxygen, the observed slight decrease in the bonding energy from $529.1 \mathrm{eV}$ for BSCF to $529.0 \mathrm{eV}$ for BKSCF suggests a weaker TM-O bond and a more active oxygen atom in $\mathrm{BKSCF}$ compared with $\mathrm{BSCF},{ }^{53}$ which could accelerate the ORR reactions.

The reported experimental and theoretical findings were encouraging and pushed us to test BKSCF in H-SOFC measurements. Figure 5 shows typical SEM micrographs of the H-SOFCs with a BKSCF cathode. The cell exhibits a four-layer structure consisting of the anode substrate, the anode functional layer, the electrolyte, and the cathode. The dense electrolyte membrane contact with both the cathode and 
the anode functional layer is good. The anode functional layer was used to improve the contact between the electrolyte and the anode. ${ }^{24}$ Compared with the relatively dense anode functional layer, some big pores created by the pore former (starch) can be observed at the anode support. The thickness of the cathode, electrolyte, anode functional layer and anode support is about $20 \mu \mathrm{m}, 10 \mu \mathrm{m}, 20 \mu \mathrm{m}$ and $0.6 \mathrm{~mm}$, respectively. The cell using a BSCF cathode with a similar microstructure was also fabricated for comparison (Figure S2).

Figure 6(a) shows the I-V and power density curves of H-SOFCs using a BKSCF cathode. For comparison, the fuel cell performance of the cell using the BSCF cathode is shown in Figure 6(b). The open circuit voltage (OCV) for the BSCF cell was $1,0.99$ and $0.98 \mathrm{~V}$ at 600,650 and $700{ }^{\circ} \mathrm{C}$, respectively, while the OCV for the BKSCF cell was $1.02,1.01$ and $0.99 \mathrm{~V}$ at 600,650 and $700{ }^{\circ} \mathrm{C}$, respectively. The OCV values are quite close for these two cells, but they are still lower than the theoretical values that reach $1.05 \mathrm{~V}$ at $700{ }^{\circ} \mathrm{C} .{ }^{5}$ The slightly lower measured OCV compared with the theoretical one is probably due to the appearance of some hole conductions for proton-conducting oxides at high temperatures. ${ }^{54}$ The peak power density for the cell using the BSCF cathode reached 289, 480 and $904 \mathrm{~mW} \mathrm{~cm}^{-2}$ at 600, 650 and $700{ }^{\circ} \mathrm{C}$, respectively. A fuel cell performance improvement was obtained by changing the cathode from BSCF to BKSCF, and the peak power density for the cell using the BKSCF cathode reached 441,737 and $1275 \mathrm{~mW} \mathrm{~cm}^{-2}$ at 600 , 650 and $700{ }^{\circ} \mathrm{C}$, respectively. Table 4 summarizes the fuel cell performance of 
H-SOFCs using the BSCF-based cathode in the literature and in this study. One can see that the BSCF cathode only shows moderate performance for H-SOFCs. With microstructure optimization (such as the reduction of electrolyte thickness or the use of an electrode functional layer), the fuel cell performance is obviously improved. Further and dramatic cell performance improvement was obtained by using the BKSCF, thus reaching a high performance for the BSCF-based cathode for H-SOFCs. Despite the fact that making direct comparisons for SOFC tests is difficult due to the variability of many parameters, the cell using a BKSCF cathode, as reported in this study, showed a much larger power output than most of H-SOFCs using a BSCF-based cathode reported previously, ${ }^{18,24,55-59}$ which suggests that K-doping into BSCF can enhance the cathode catalytic activity, thus allowing a fuel cell performance improvement. The only exception is reported by An et al. ${ }^{60}$ In their study, proton-conducting SOFCs using single-phase BSCF cathode were fabricated. Although BSCF shows a big thermal expansion mismatch with BCZY-based electrolyte, their unique fabrication procedure seems possible to solve the problem and makes the use of single phase BSCF cathode for BCZY cells with any delamination. In addition, a very thin electrolyte (about $4 \mu \mathrm{m}$ ) used in their study could be another reason for the high fuel cell performance, suggesting the optimization of the cell structure would be a feasible approach to further improve the cell performance. Furthermore, the cathode/electrolyte interface engineering, proposed by Choi et $a l^{6}$ by fabricating a thin layer of proton-electron mixed 
conductor $\mathrm{PrBa}_{0.5} \mathrm{Sr}_{0.5} \mathrm{Co}_{1.5} \mathrm{Fe}_{0.5} \mathrm{O}_{5+\delta}$ to promote the interfacial reactions, is another interesting route to enhance the cell performance.

Figure 7 (a) shows the EIS plots of the cells measured at $600{ }^{\circ} \mathrm{C}$. The intercept at a high-frequency with the real-axis represents the ohmic resistance of the cell $\left(R_{\text {ohmic }}\right)$, while the difference between the high- and low-frequency intercepts is the polarization resistance of the cell $\left(R_{p}\right)$. One can see that the $R_{\text {ohmic }}$ was 0.23 and 0.26 $\Omega \mathrm{cm}^{2}$ at $600{ }^{\circ} \mathrm{C}$ for cells using the BKSCF and BSCF cathodes, respectively. In contrast to the close $R_{\text {ohmic }}$, the $R_{p}$ differs a lot for these two cells. The $R_{p}$ for the cell using BSCF cathode was $0.55 \Omega \mathrm{cm}^{2}$ at $600{ }^{\circ} \mathrm{C}$, while the corresponding value for the cell using BKSCF cathode was $0.33 \Omega \mathrm{cm}^{2}$ at the same testing temperature. The $R_{p}$ for the cell using BKSCF decreased to $0.054 \Omega \mathrm{cm}^{2}$ at $700{ }^{\circ} \mathrm{C}$, while the corresponding value for the cell using BSCF was $0.088 \Omega \mathrm{cm}^{2}$ at the same testing temperature (Figure 7b). As both cells, including the anode, anode functional layer and electrolyte, were fabricated in the same way, the big difference in $R_{p}$ can be ascribed mainly to the different cathode used. The improved proton migration in BKSCF, coupled with the weaker TM-O bond, improves the cathode catalytic activity as expected, thus accelerating the cathode reactions and leading to a lower $R_{p}$.

It is noted that chemical stability is an issue for BSCF material and the doping of $\mathrm{K}$ in $\mathrm{BSCF}$ that increases the basicity of the material may make the material more reactive with acid gases, such as $\mathrm{CO}_{2}$. In order to evaluate the influence of K-doping on the stability of BSCF, both BSCF and BKSCF powders were treated in $\mathrm{CO}_{2}$ and 
FTIR was used to check the materials before and after the treatment. One can see from Figure S3 that no detectable carbonate peak can be observed for BSCF and BKSCF before $\mathrm{CO}_{2}$ treatment, suggesting no formation of carbonate for the as-prepared powders. However, obvious carbonate signal can be detected for the powders after the $\mathrm{CO}_{2}$ treatment, suggesting both BSCF and BKSCF can react with $\mathrm{CO}_{2}$ forming the carbonates. $\mathrm{CO}_{2}$ TPD results (Figure $\mathrm{S} 4$ ) show that the intensity of $\mathrm{CO}_{2}$ signal for $\mathrm{BKSCF}$ is higher than that for $\mathrm{BSCF}$, indicating the doping of $\mathrm{K}$ in BSCF makes this material more reactive with $\mathrm{CO}_{2}$. This result is somehow expected as the partial replacement of $\mathrm{Ba}$ by $\mathrm{K}$ could increase the basicity of the material, thus making it more reactive with acid gases such as $\mathrm{CO}_{2}$. The BKSCF cell was tested for about $23 \mathrm{~h}$ at $600{ }^{\circ} \mathrm{C}$ with an applied voltage of $0.75 \mathrm{~V}$. The cell showed a stable performance in the first $10 \mathrm{~h}$ and the performance dropped about $20 \%$ in the rest of operation time reaching a current density of $342 \mathrm{~mA} \mathrm{~cm}^{-2}$, as shown in Figure 8. Even decreasing about $20 \%$ of the initial performance after 23 hours' operation, the performance is still larger than that for BSCF cell tested in our study which only reached a current density of $279 \mathrm{~mA} \mathrm{~cm}^{-2}$ at same testing temperature with the same applied voltage. The decreased performance might be due to the reaction between BKSCF cathode with $\mathrm{H}_{2} \mathrm{O}$ and $\mathrm{CO}_{2}$ during the fuel cell working condition.

\section{Conclusions}

$\mathrm{K}$ was used to partially replace $\mathrm{Ba}$ in the BSCF to increase the hydration property 
of the material and, thus, improve proton migration, with the aim of integrating the high catalytic activity of BSCF with proton-conduction for a better application in proton-conducting SOFCs. Both experimental and theoretical studies indicated that the doping of $\mathrm{K}$ in BSCF favoured hydration and improved proton migration. The direct assessment with experimental measurements of the proton conductivity for BSCF materials is hindered by their instability in reducing environments. To avoid this problem, DFT calculations were needed to predict the proton mobility, and the proposed successful methodology may be a general solution for similar problems. In addition, K-doping weakened the O-TM bond, thus making the oxygen atom more active, which benefited the cathode reactions. As a result, the cell using a BKSCF cathode showed an impressive fuel cell output. The current study provides a rational way to design of a cathode material with high performance for proton-conducting SOFCs, which might be applied in a broader area for introducing proton migrations in the material.

\section{Conflicts of interest}

There are no conflicts to declare.

\section{Acknowledgements}


This work was supported by the National Natural Science Foundation of China (Grant No.: 51602238), the Natural Science Foundation of Shandong Province (Grant No.: ZR2018JL017).

\section{Notes and references}

[1] A.B. Stambouli, E. Traversa, Renew. Sust. Energy Rev., 2002, 6, 433-455.

[2] E. Wachsman, T. Ishihara, J. Kilner, Mrs Bull., 2014, 39, 773-782.

[3] E. Fabbri, L. Bi, D. Pergolesi, E. Traversa, Adv. Mater., 2012, 24, 195-208.

[4] L. Bi, S. Boulfrad, E. Traversa, Chem. Soc. Rev., 2014, 43, 8255-8270.

[5] E. Fabbri, L. Bi, H. Tanaka, D. Pergolesi, E. Traversa, Adv. Funct. Mater., 2011, 21, 158-166.

[6] S. Choi, C.J. Kucharczyk, Y.G. Liang, X.H. Zhang, I. Takeuchi, H.I. Ji, S.M. Haile, Nature Energy, 2018, 3, 202-210.

[7] E. Fabbri, L. Bi, D. Pergolesi, E. Traversa, Energy Environ. Sci., 2011, 4, 4984-4993.

[8] M.R. Li, M.W. Zhao, F. Li, W. Zhou, V.K. Peterson, X.Y. Xu, Z.P. Shao, I. Gentle, Z.H. Zhu, Nature Commun., 2017, 8.

[9] F. He, T.Z. Wu, R.R. Peng, C.R. Xia, J. Power Sources, 2009, 194, 263-268.

[10] X.F. Ding, Z.P. Gao, D. Ding, X.Y. Zhao, H.Y. Hou, S.H. Zhang, G.L. Yuan, Appl. Catal. B Environ., 2019, 243, 546-555.

[11] E. Fabbri, D. Pergolesi, E. Traversa, Sci. Technol. Adv. Mater., 2010, 11, 044301. 
[12] E. Fabbri, T.K. Oh, S. Licoccia, E. Traversa, E.D. Wachsman, J. Electrochem. Soc., 2009, 156, B38-B45.

[13] R.R. Peng, T.Z. Wu, W. Liu, X.Q. Liu, G.Y. Meng, J. Mater. Chem., 2010, 20, 6218-6225.

[14] Z.P. Shao, S.M. Haile, Nature, 2004, 431, 170-173.

[15] R. Zohourian, R. Merkle, G. Raimondi and J. Maier, Adv. Funct. Mater., 2018, 28, 1801241.

[16] A. Grimaud, F. Mauvy, J.M. Bassat, S. Fourcade, L. Rocheron, M. Marrony, J.C. Grenier, J. Electrochem. Soc., 2012, 159, B683-B694.

[17] S.C. Sun, Z. Cheng, J. Electrochem. Soc., 2017, 164, F3104-F3113.

[18] R.R. Peng, Y. Wu, L.Z. Yang, Z.Q. Mao, Solid State Ionics, 2006, 177, 389-393.

[19] W. Zhou, R. Ran, Z.P. Shao, J. Power Sources, 2009, 192, 231-246.

[20] E. Fabbri, D. Pergolesi, E. Traversa, Chem. Soc. Rev., 2010, 39, 4355-4369.

[21] K.D. Kreuer, Annu. Rev. Mater. Res., 2003, 33, 333-359.

[22] L. Bi, S.P. Shafi, E.H. Da'as, E. Traversa, Small, 2018, 14, 1801231.

[23] E.H. Da'as, L. Bi, S. Boulfrad, E. Traversa, Sci. China Mater., 2018, 61, 57-64.

[24] L. Bi, E. Fabbri, E. Traversa, Electrochem. Commun., 2012, 16, 37-40.

[25] P. Hohenberg, W. Kohn, Phys. Rev., 1964, 136, B864.

[26] P.E. Blöchl, O. Jepsen, O.K. Andersen, Phys. Rev. B, 1994, 49, 16223.

[27] G. Kresse, J. Furthmuller, Phys. Rev. B, 1996, 54, 11169-11186.

[28] M.C. Payne, M.P. Teter, D.C. Allan, T. Arias, A.J. Joannopoulos, Rev. Modern 
Phys., 1992, 64, 1045.

[29] W. Kohn, L.J. Sham, Phys. Rev., 1965, 140, A1133.

[30] G. Kresse, D. Joubert, Phys. Rev. B, 1999, 59, 1758.

[31] V.I. Anisimov, J. Zaanen, O.K. Andersen, Phys. Rev. B, 1991, 44, 943.

[32] V.I. Anisimov, F. Aryasetiawan, A. Lichtenstein, J. Phys.: Conden. Matt., 1997, 9, 767.

[33] C. Zhang, P.D. Bristowe, RSC Adv., 2013, 3, 12267-12274.

[34] G. Henkelman, B.P. Uberuaga, H. Jónsson, J. Chem. Phys., 2000, 113, 9901-9904.

[35] Shannon Radii, http://abulafia.mt.ic.ac.uk/shannon/ptable.php

[36] T. Norby, M. Wideroe, R. Glockner, Y. Larring, Dalton Trans., 2004, 3012-3018.

[37] Y.A. Mastrikov, M.M. Kuklja, E.A. Kotomin, J. Maier, Energy Environ. Sci., 2010, 3, 1544-1550.

[38] A.B. Munoz-Garcia, M. Pavone, Chem. Mater., 2016, 28, 490-500.

[39] A.M. Saranya, D. Pla, A. Morata, A. Cavallaro, J. Canales-Vazquez, J.A. Kilner, M. Burriel, A. Tarancon, Adv. Energy Mater., 2015, 5.

[40] L. Yang, Z. Liu, S.Z. Wang, Y.M. Choi, C.D. Zuo, M.L. Liu, J. Power Sources, $2010,195,471-474$.

[41] C. Su, X.M. Xu, Y.B. Chen, Y. Liu, M.O. Tade, Z.P. Shao, J. Power Sources, 2015, 274, 1024-1033. 
[42] H.M. Zhang, B.A. Wilhite, J. Membr. Sci., 2016, 512, 104-110.

[43] D.L. Han, S. Uemura, C. Hiraiwa, M. Majima, T. Uda, Chemsuschem, 2018, 11, $4102-4113$.

[44] A. Endo, M. Ihara, H. Komiyama and K. Yamada, Solid State Ionics, 1996, 86-8, 1191-1195.

[45] G. L. Xiao, Q. A. Liu, F. Zhao, L. Zhang, C. R. Xia and F. L. Chen, J. Electrochem. Soc., 2011, 158, B455-B460.

[46] R. A. Huggins, Ionics, 2002, 8, 300-313

[47] A.B. Munoz-Garcia, M. Tuccillo, M. Pavone, J. Mater. Chem. A, 2017, 5, $11825-11833$.

[48] A.B. Munoz-Garcia, M. Pavone, Int. J. Quantum Chem., 2016, 116, 1501-1506.

[49] Z.Q. Wang, W.Q. Yang, S.P. Shafi, L. Bi, Z.B. Wang, R.R. Peng, C.R. Xia, W.

Liu, Y.L. Lu, J. Mater. Chem. A, 2015, 3, 8405-8412.

[50] J. Kim, S. Sengodan, G. Kwon, D. Ding, J. Shin, M.L. Liu, G. Kim, Chemsuschem, 2014, 7, 2811-2815.

[51] L.P. Sun, H. Li, Q. Li, L.H. Huo, H. Zhao, J.M. Bassat, M. Rougier, S. Fourcade, J.C. Grenier, J. Power Sources, 2018, 392, 8-14.

[52] Z. X. Wang, Z. Q. Yang, Y. F. Song, J. K. Mao, F. L. Liang and W. Zhou, Int. J. Hydrogen Energy, 2018, 43, 13420-13429.

[53] Q. Zeng, Y. Zuo, C. Fan, C. Chen, J. Membr. Sci., 2009, 335, 140-144.

[54] G. L. Ma, H. Matsumoto and H. Iwahara, Solid State Ionics, 1999, 122, 237-247. 
[55] Y. Lin, R. Ran, Y. Zheng, Z. Shao, W. Jin, N. Xu, J. Ahn, J. Power Sources, $2008,180,15-22$.

[56] Y. Guo, Y. Lin, R. Ran, Z. Shao, J. Power Sources, 2009, 193, 400-407.

[57] B. Guan, Z. Lu, G. Wang, B. Wei, W. Li, X. Huang, Fuel Cells, 2012, 12, $141-145$.

[58] T.-T. Wan, A.-K. Zhu, H.-B. Li, C.-C. Wang, Y.-M. Guo, Z.-P. Shao, O.

Savadogo, Rare Metals, 2018, 37, 633-641.

[59] Y. Xie, N. Shi, D. Huan, W. Tan, J. Zhu, X. Zheng, H. Pan, R. Peng, C. Xia, Chemsuschem, 2018, 11, 3423-3430.

[60] H. An, H. W. Lee, B. K. Kim, J. W. Son, K. J. Yoon, H. Kim, D. Shin, H. I. Ji and J. H. Lee, Nature Energy, 2018, 3, 870-875. 

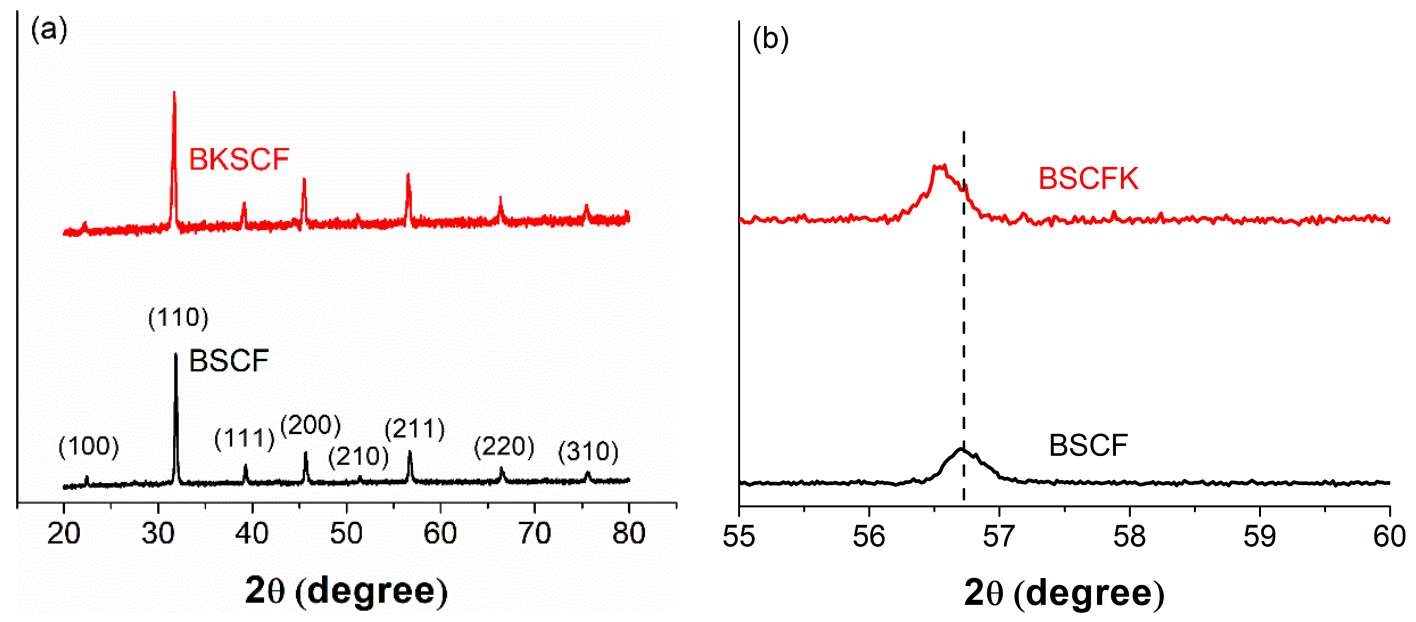

Figure 1. XRD patterns for (a)BSCF and BKSCF powders; (b)Enlarged reflection peak of (211) plane for the samples 


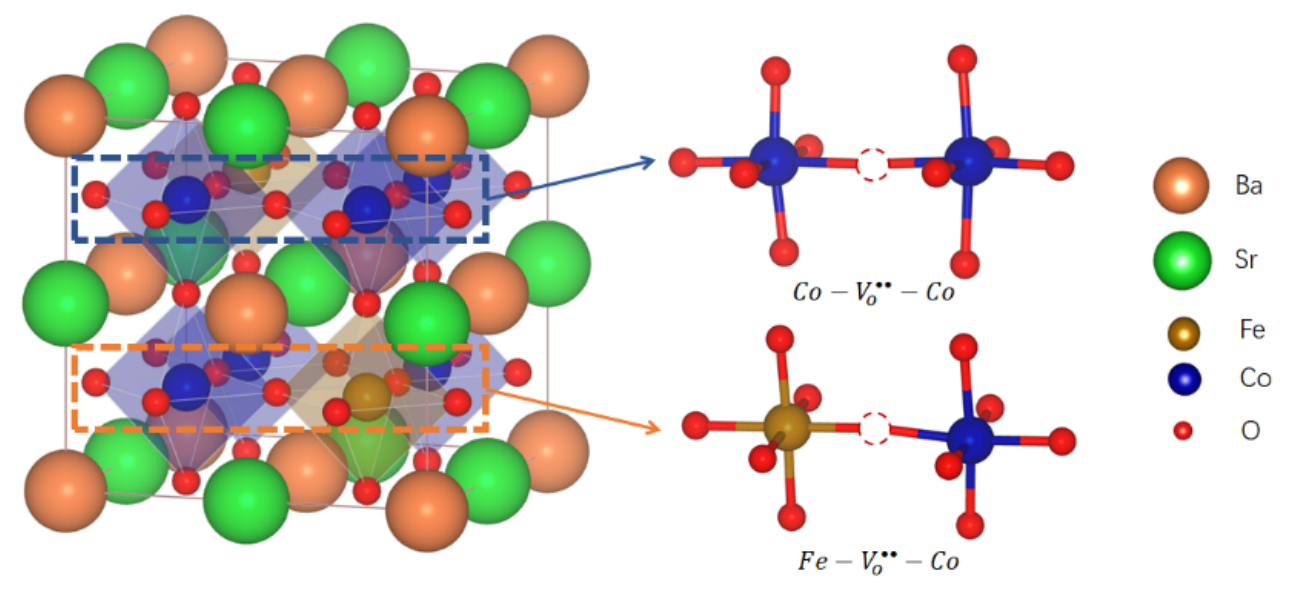

Figure 2. Scheme of Co- $V_{o}^{\cdot \bullet}$-Co and Fe- $V_{o}^{\cdot \bullet}$-Co vacancies. 


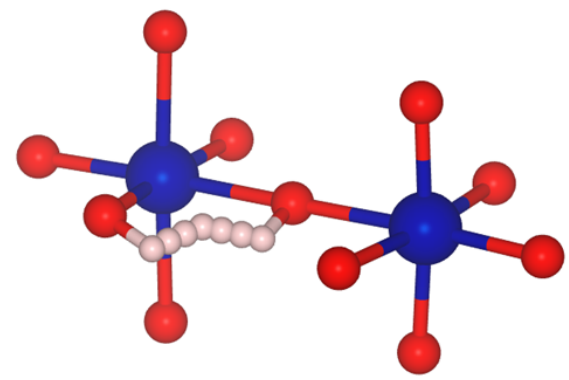

Hopping step

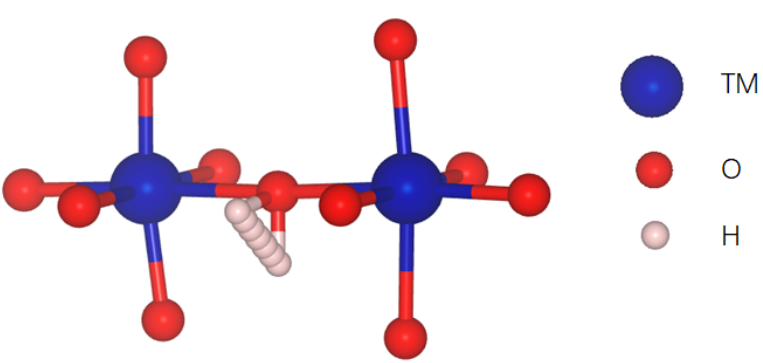

Rotating step

Figure 3. Scheme of the hopping and rotating steps for proton migration. 

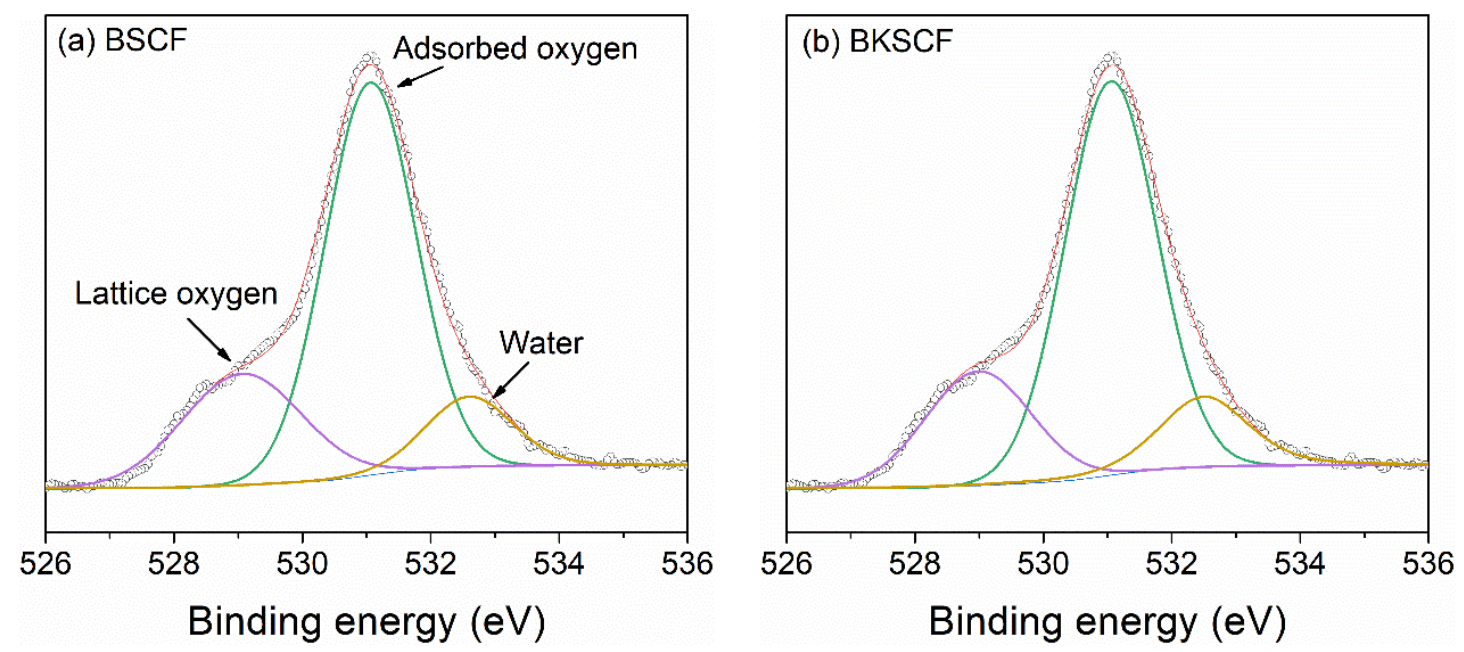

Figure 4. Ols binding energy curves for BSCF (a) and BKSCF (b). 

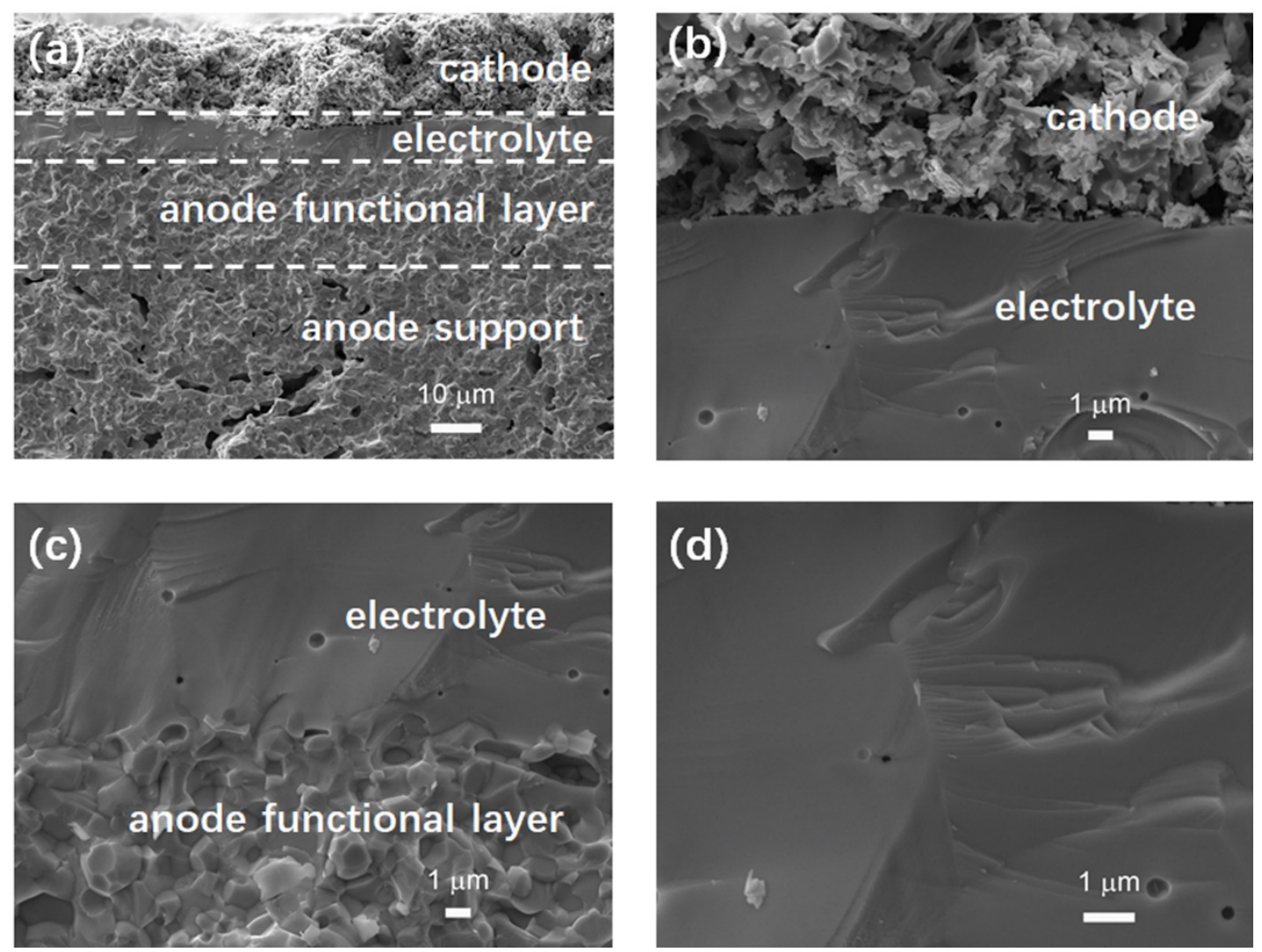

Figure 5. Cross-sectional SEM micrographs of the cell using BKSCF cathode: (a) the complete cell; (b) cathodelelectrolyte; (c) electrolyte/anode functional layer; (d) electrolyte. 

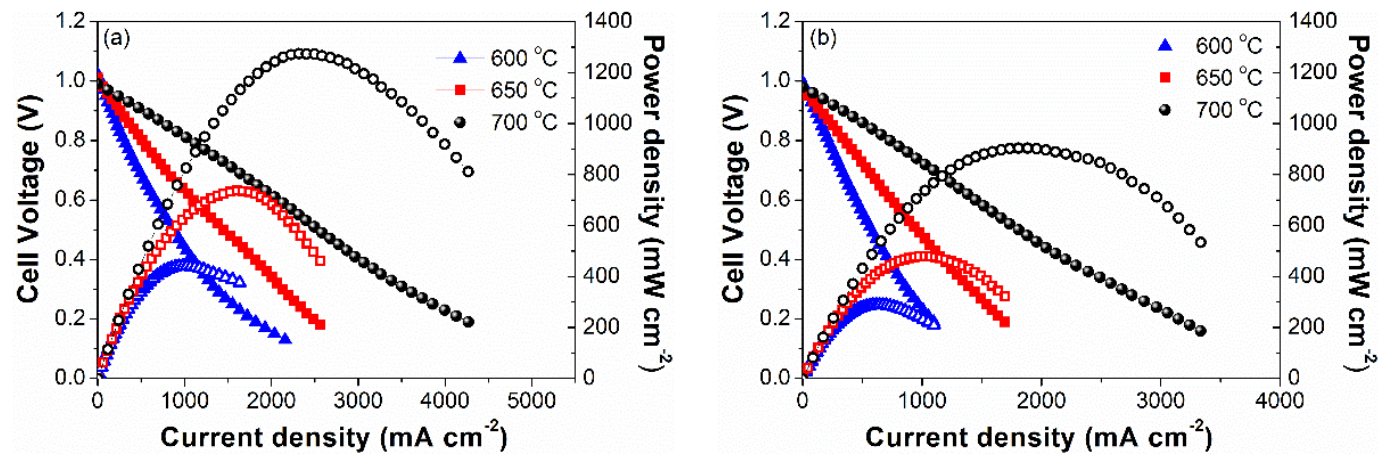

Figure 6. I-V and power density curves for the cell using (a) BKSCF cathode and (b) BSCF cathode. 

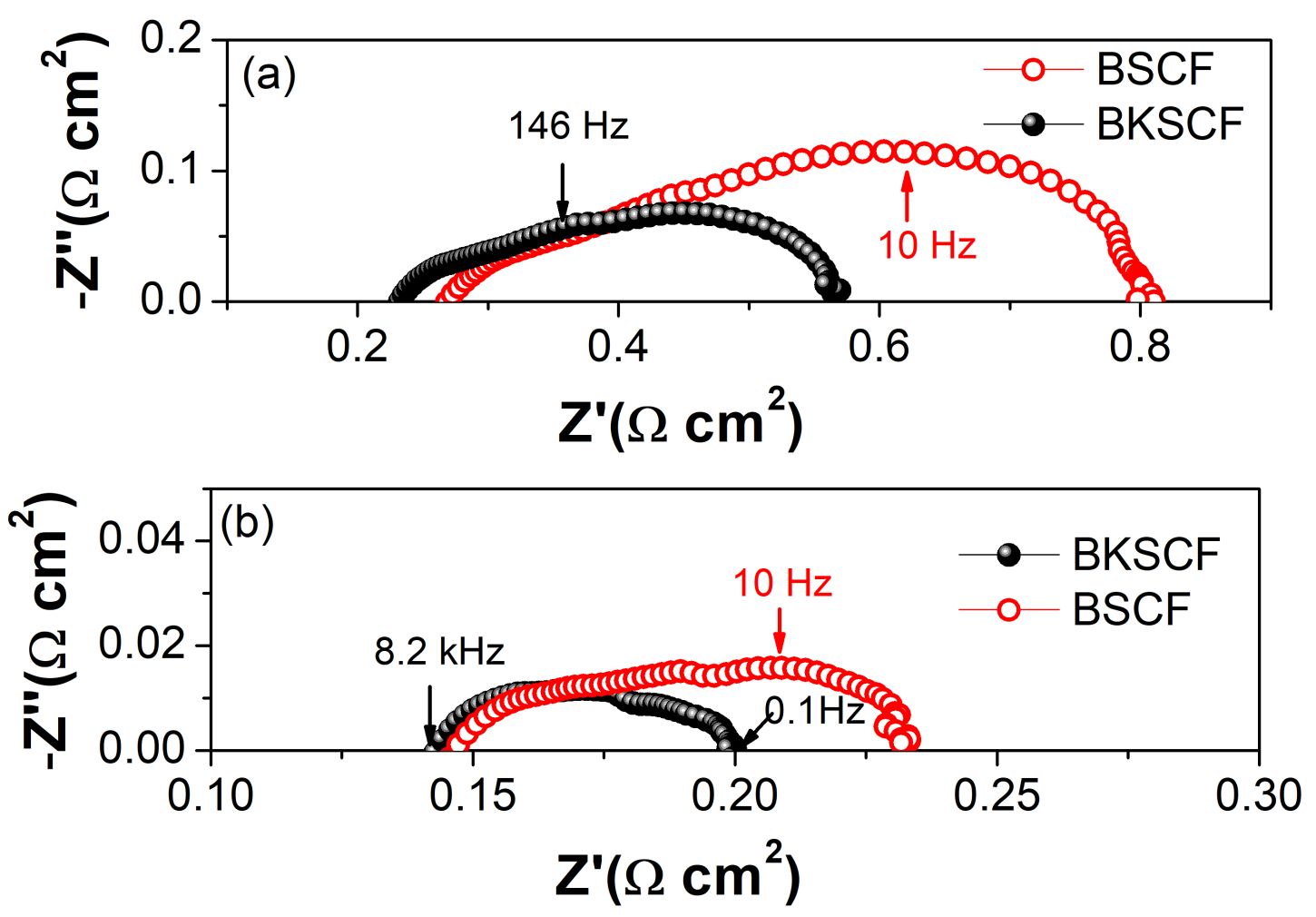

Figure 7. EIS plots of the cell using BSCF and BKSCF cathode tested at (a) $600^{\circ} \mathrm{C}$ and (b) $700^{\circ} \mathrm{C}$. 


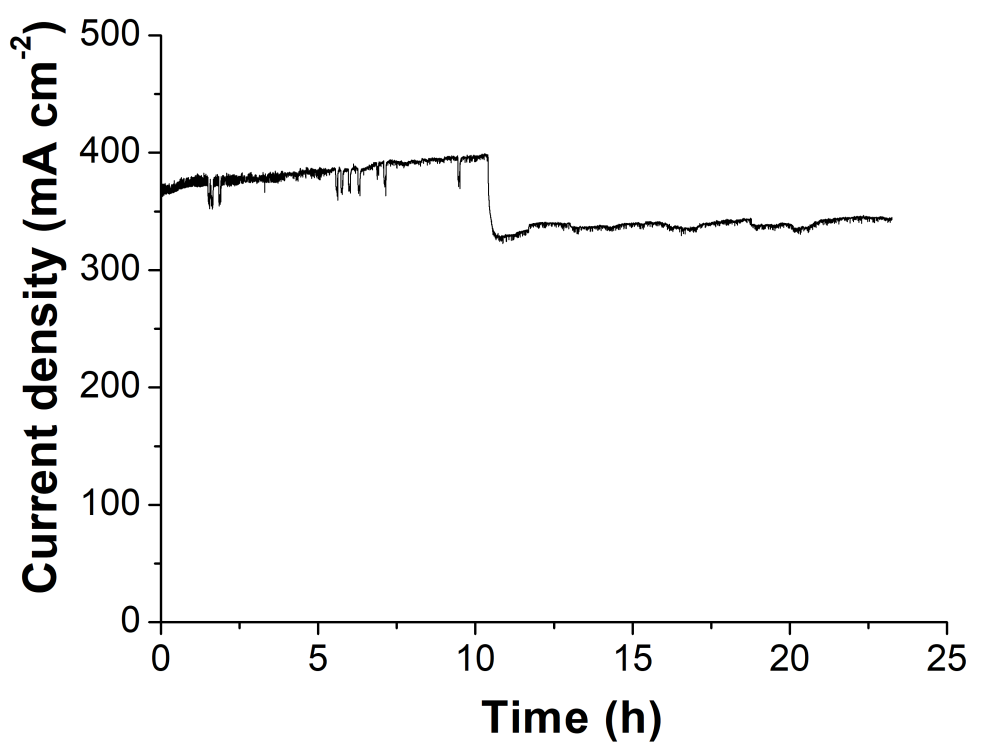

Figure 8 . The current density of a BKSCF cell as a function of working time under an applied voltage of $0.75 \mathrm{~V}$. 
Table 1. Oxygen vacancy formation energy $\left(E_{f}\right)$ of Co- $V_{o}^{\cdot \bullet}$-Co and Fe- $V_{o}^{\cdot \bullet}$-Co in BSCF and $B K S C F$.

\begin{tabular}{|l|l|l|}
\hline & $E_{f}\left(C o-V_{o}^{\cdot \bullet}-C o\right) / \mathrm{eV}$ & $E_{f}\left(F e-V_{o}^{*}-C o\right) / \mathrm{eV}$ \\
\hline BSCF & -1.19 & -1.21 \\
\hline BKSCF & -1.69 & -1.51 \\
\hline
\end{tabular}

Table 2. Hydration energy in BSCF and BKSCF

\begin{tabular}{|l|l|l|}
\hline & $\Delta E_{\text {hydration }}\left(C o-V_{o}^{*}-C o\right) / \mathrm{eV}$ & $\Delta E_{\text {hydration }}\left(F e-V_{o}^{*}-C o\right) / \mathrm{eV}$ \\
\hline BSCF & -0.04 & -0.21 \\
\hline BKSCF & -0.21 & -0.3 \\
\hline
\end{tabular}

Table 3. Hopping and rotating energy values for proton migration in BSCF and BKSCF.

\begin{tabular}{|l|l|l|}
\hline & Hopping energy $(\mathrm{eV})$ & Rotating energy $(\mathrm{eV})$ \\
\hline BSCF & 0.22 & 0.33 \\
\hline BKSCF & 0.34 & 0.07 \\
\hline
\end{tabular}


Table 4. Performance of proton-conducting SOFCs using BSCF-based cathodes reported in the literature and in this study. BCY10: $\mathrm{BaCe}_{0.9} \mathrm{Y}_{0.1} \mathrm{O}_{3-\delta} ; \mathrm{BCZY442:} \mathrm{BaCe}_{0.4} \mathrm{Zr}_{0.4} \mathrm{Y}_{0.2} \mathrm{O}_{3-\delta} ; \mathrm{BCM}$ : $\mathrm{BaCe}_{0.8} \mathrm{Sm}_{0.2} \mathrm{O}_{3-\delta} ; \mathrm{BCY} 20: \mathrm{BaCe}_{0.8} \mathrm{Y}_{0.2} \mathrm{O}_{3-\delta} ; \mathrm{BCZY-ZnO}: \mathrm{BaCe} e_{0.7} \mathrm{Zr}_{0.1} \mathrm{Y}_{0.2} \mathrm{O}_{3-\delta}$ with $\mathrm{ZnO}$ sintering aid; $B C Z Y: B a C e_{0.7} Z r_{0.1} Y_{0.2} O_{3-\delta} ; B C M F: B a C e_{0.8} \mathrm{Sm}_{0.2} F_{0.1} O_{3-\delta} ; B C Z Y 3: B a C e_{0.55} Z_{0.3} Y_{0.15} O_{3-\delta}$; N/A: not available.

\begin{tabular}{|l|l|l|l|}
\hline Reference & $\begin{array}{l}\text { Electrolyte } \\
\text { composition } \\
\text { thickness }(\mu \mathrm{m})\end{array}$ & $\begin{array}{l}\text { Anode } \\
\text { composition }\end{array}$ & $\begin{array}{l}\text { Power density at } 700 \\
{ }^{\circ} \mathrm{C}\left(\mathrm{mW} \mathrm{cm}^{-2}\right)\end{array}$ \\
\hline 18 & BSM, 65 & NiO+BCY10 & 340 \\
\hline 24 & BCZY442, 20 & NiO+BCZY442 & 281 \\
\hline 55 & BCY10, 20 & NiO+BSM & 550 \\
\hline 56 & BCY20, 30 & NiO+BCY20 & 500 \\
\hline 57 & BCZY-ZnO, N/A & NiO+BCZY-ZnO & 536 \\
\hline 58 & BCZY, N/A & BCZY+NiO & 602 \\
\hline 59 & BSMF, 15 & BSMF+NiO & 480 \\
\hline 60 & BCZY3, 4 & BCZY3+NiO & $1300\left(\right.$ at $\left.600{ }^{\circ} \mathrm{C}\right)$ \\
\hline This study (BSCF) & BCZY, 10 & BCZY+NiO & 904 \\
\hline This study (BKSCF) & BCZY, 10 & BCZY+NiO & 1275 \\
\hline
\end{tabular}

